\title{
Editorial: The 2018 François Naftali Frenkiel Award for Fluid Mechanics
}

The recipients of the 35th François Naftali Frenkiel Award for Fluid Mechanics are Alberto Villois, Davide Proment, and Giorgio Krstulovic for their paper "Universal and nonuniversal aspects of vortex reconnections in superfluids" which was published in Phys. Rev. Fluids 2, 044701 (2017). They were presented with this award on November 20, 2018 at the annual meeting of the APS Division of Fluid Dynamics in Atlanta, Georgia. They each received a check for $\$ 500$ plus a scroll acknowledging the award. The Award honors their innovative use of theory and numerical simulations to examine reconnection events in various superfluid vortex configurations. The results provide new understanding of universality and irreversibility of superfluid vortex connections. A superfluid vortex is an example of a topological defect and their approach could also be applicable to other physical systems where topological defects arise.

The purpose of this Award, which is named after Dr. F. N. Frenkiel, founder and longtime editor of Physics of Fluids, from which Physical Review Fluids evolved, is to recognize significant contributions in fluid mechanics by young investigators. The Award is sponsored by the Division of Fluid Dynamics of the APS and beginning with the 2017 award, is given annually to a young author of a paper that has been published in Physical Review Fluids, during the calendar year preceding the presentation. "Young" will normally be defined as being under 40 years of age. More than one author may be involved, and one author may be a thesis advisor sharing the Award if he/she is also under 40. The winner of this Award is selected by a committee appointed by the Chair of the Division of Fluid Dynamics of the APS.

François Naftali Frenkiel was born in Warsaw, Poland on September 19, 1910, and received his Ph.D. in Physics from the University of Lille in France, where he studied under the direction of Kampé de Fériet. He came to the U.S. in 1947 and was associated successively with Cornell University, the U.S. Naval Ordnance Laboratory, the Johns Hopkins University Applied Physics Laboratory, and, from 1960 until his retirement, with the David W. Taylor Naval Ship Research and Development Center. In addition to being the founder and longtime editor of Physics of Fluids, he served on a large number of national and international committees, including the Division of Fluid Dynamics of the American Physical Society, of which he was the chairman and secretary on numerous occasions. $\mathrm{He}$ published extensively in the field of turbulent flows and pioneered the application of high-speed digital computing methods to the measurement of turbulence and the mathematical modeling of urban pollution. Dr. Frenkiel retired in 1981 and passed away on July 9, 1986, in Washington, DC. 


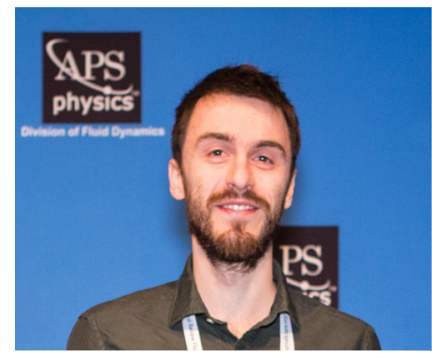

Alberto Villois received his Ph.D. in Applied Mathematics at the University of East Anglia (Norwich, UK) in 2017 and he is currently a postdoctoral researcher at the University of Bath, UK. His research interests cover solitons theory, out-of-equilibrum quantum fluids and dynamics of topological defects.

Davide Proment was awarded his Ph.D. in Physics in 2011 at the Università degli Studi di Torino (Turin, Italy) supervised by Prof. Miguel Onorato, with whom he worked later as a postgraduate research assistant. In November 2012 he was appointed Lecturer at the School of Mathematics of the University of East Anglia (Norwich, United Kingdom), where he currently works as a Senior Lecturer. Davide's past and present research interests focus mainly on vortex dynamics, wave interactions, and turbulence in superfluids. More generally, he is interested in nonlinear physics, statistical mechanics and out-of-equilibrium systems.

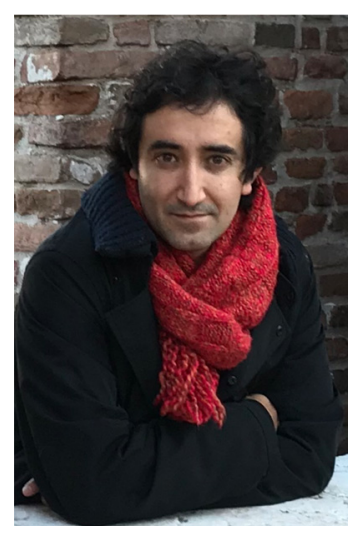

Giorgio Krstulovic obtained his Ph.D. in 2010 at Laboratoire de Physique Statistique (ENS Paris- Paris VI). Thanks to a CNRS-ERC postdoc fellowship he then moved to Observatoire de la Côte d'Azur (OCA) in Nice, France, to work on particle transport and cloud physics. Since 2013 he is a CNRS permanent researcher at OCA. His present research interests are mainly hydrodynamic turbulence, particle transport by turbulent flows, vortex dynamics, wave turbulence, and superfluid dynamics. In general, he is interested in statistical mechanics, nonlinear science and theoretical aspects of turbulence.

John Kim

Gary Leal

Editors

\author{
Published 31 January 2019
}

DOI: 10.1103/PhysRevFluids.4.010002 